PROCEEDINGS OF THE

AMERICAN MATHEMATICAL SOCIETY

Volume 134, Number 5, Pages 1315-1317

S 0002-9939(05)08416-9

Article electronically published on October 25, 2005

\title{
CHARACTERIZATION OF QUASI-BANACH SPACES WHICH COARSELY EMBED INTO A HILBERT SPACE
}

\author{
N. LOVASOA RANDRIANARIVONY
}

(Communicated by David Preiss)

\begin{abstract}
We show that a quasi-Banach space coarsely embeds into a Hilbert space if and only if it is isomorphic to a linear subspace of $L_{0}(\mu)$ for some probability space $(\Omega, \mathcal{B}, \mu)$.
\end{abstract}

A (not necessarily continuous) map $f$ between two metric spaces $(X, d)$ and $(Y, \delta)$ is called a coarse embedding (see [G, 7.G]) if there exist two nondecreasing functions $\varphi_{1}:[0, \infty) \rightarrow[0, \infty)$ and $\varphi_{2}:[0, \infty) \rightarrow[0, \infty)$ such that

(1) $\varphi_{1}(d(x, y)) \leq \delta(f(x), f(y)) \leq \varphi_{2}(d(x, y))$,

(2) $\varphi_{1}(t) \rightarrow \infty$ as $t \rightarrow \infty$.

It was proved in JR that $\ell_{p}$ does not coarsely embed into a Hilbert space when $p>2$. The present article is a strengthening of that result by giving a full characterization of quasi-Banach spaces that coarsely embed into a Hilbert space. This result, as well as its proof, mirrors the theorem in AMM which characterizes spaces that uniformly embed into a Hilbert space. The combination of Theorem 1 and the theorem in AMM yield that a quasi-Banach space uniformly embeds into a Hilbert space if and only if it coarsely embeds into a Hilbert space. This is counterintuitive in that a uniform embedding gives information only on small distances while a coarse embedding gives information only on large distances.

Theorem 1. A quasi-Banach space $X$ coarsely embeds into a Hilbert space if and only if there is a probability space $(\Omega, \mathcal{B}, \mu)$ such that $X$ is linearly isomorphic to a subspace of $L_{0}(\mu)$.

To prove Theorem 1, we use a result essentially contained in [JR as is recalled in the following proposition.

Proposition 2. Let $X$ be a quasi-Banach space which coarsely embeds into a Hilbert space. Then there exists on $X$ a continuous negative definite function $g$ which satisfies $g(0)=0$ and $\phi_{1}(\|x\|) \leq g(x) \leq\|x\|^{2 \alpha}$ where $\phi_{1}:[0, \infty) \rightarrow[0, \infty)$ is a nondecreasing function satisfying $\phi_{1}(t) \rightarrow \infty$ as $t \rightarrow \infty$, and $\alpha>0$.

Received by the editors November 17, 2004.

2000 Mathematics Subject Classification. Primary 46B20; Secondary 51F99.

Key words and phrases. Coarse embedding, uniform embedding.

The author was supported in part by NSF 0200690 and Texas Advanced Research Program 010366-0033-20013.

This paper represents a portion of the author's dissertation being prepared at Texas A\&M University under the direction of William B. Johnson.

(C) 2005 by the author 
Proof. Steps 0, 1, 2 (and a piece of Step 3 on the continuity of $g$ ) in JR extend to the case of quasi-Banach spaces.

Proof of Theorem 1. Let $X$ be a quasi-Banach space. A theorem of Aoki and Rolewicz ( $\underline{\mathrm{A}}$ and $[\underline{\mathrm{R}})$ gives an equivalent quasi-norm $\|\cdot\|$ on $X$ which is also $p$-subadditive for some $0<p \leq 1$, i.e., $\|x+y\|^{p} \leq\|x\|^{p}+\|y\|^{p}$ for all $x, y$ in $X$. In particular, $X$ under this norm has type $p$.

Say $X$ is linearly isomorphic to a subspace of $L_{0}(\mu)$ for some probability space $(\Omega, \mathcal{B}, \mu)$. Then since $X$ has type $p, X$ is isomorphic to a subspace of $L_{r}(\mu)$ for every $r<p$. (See [Ni] and also [BL, Theorem 8.15].)

Now since $r<2$, Nowak's result in $[\mathrm{No}$ implies that $X$ coarsely embeds into a Hilbert space. In fact, Nowak notices that the negative definite function $x \in L_{r} \mapsto$ $\|x\|_{L_{r}}^{r}$ for $0<r<2$ gives, via Schoenberg's classical work [S], a coarse embedding of $L_{r}$ into a Hilbert space. This explicit representation of a negative definite function in $L_{r}$ appears in several works by Guerre-Delabrière; see GD for example. Mendel and Naor in [MN] actually give an explicit formula for a coarse embedding of $L_{r}$ into $L_{q}$ when $r<q$ given by $T: L_{r}(\mathbb{R}) \rightarrow L_{q}(\mathbb{R} \times \mathbb{R})$ :

$$
T(f)(s, t)=\frac{1-e^{i t f(s)}}{|t|^{(r+1) / q}} .
$$

Conversely, let $X$ be a quasi-Banach space which coarsely embeds into a Hilbert space. Let $g$ be the negative definite function on $X$ given by Proposition 2, and let $f$ be the continuous positive definite function given by $f=e^{-g}$. Use Lemma 4.2 in [AMM] (see also Lemma 1 in [BDK, page 238] and Proposition 8.7 in [BL]) to get a probability space $(\Omega, \mathcal{B}, \mu)$ and a continuous linear operator $U: X \rightarrow L_{0}(\mu)$ such that the characteristic function $\mathbb{E} \exp (i t U x)$ of $U x$ is equal to $f(t x)$ for every $x \in X$ and $t \in \mathbb{R}$. We show that $U$ is an isomorphism into.

Let $\left(x_{n}\right)_{n}$ be a sequence in $X$ such that $U\left(x_{n}\right) \rightarrow 0$ in $L_{0}(\mu)$, i.e., in measure. Then $f\left(t x_{n}\right)=\mathbb{E}\left(\exp \left(i t U x_{n}\right)\right) \rightarrow 1$ for each fixed $t$ in $\mathbb{R}$.

If $\left(x_{n}\right)_{n}$ does not converge to 0 , then by passing to a subsequence we can assume without loss of generality that $\left\|x_{n}\right\| \geq \epsilon$ for all $n$ and for some $\epsilon>0$. But since $\phi_{1}$ is nondecreasing, we get for every $t>0$,

$$
e^{-\phi_{1}\left(t\left\|x_{n}\right\|\right)} \leq e^{-\phi_{1}(t \epsilon)}
$$

Since $\phi_{1}(s) \rightarrow \infty$ as $s \rightarrow \infty$, we can pick $t_{0}>0$ so that $e^{-\phi_{1}\left(t_{0} \epsilon\right)}<\frac{1}{2}$. For that $t_{0}$, we have for every $n$,

$$
f\left(t_{0} x_{n}\right) \leq e^{-\phi_{1}\left(t_{0} \epsilon\right)}<\frac{1}{2} .
$$

This contradicts the fact that $f\left(t_{0} x_{n}\right) \rightarrow 1$.

Thus $x_{n} \rightarrow 0$, and hence $U$ is one-to-one and its inverse is continuous.

\section{ACKNOWLEDGEMENT}

I would like to thank William B. Johnson for the constructive conversations that led to the production of this article.

\section{REFERENCES}

[A] T. Aoki, Locally bounded linear topological spaces, Proc. Imp. Acad. Tokyo 18 (1942), 588-594. MR0014182(7:250d) 
[AMM] I. Aharoni, B. Maurey, and B. S. Mityagin, Uniform embeddings of metric spaces and of Banach spaces into Hilbert spaces, Israel J. Math. 52 (1985), no. 3, 251-265. MR0815815 (87b:46011)

[BDK] J. Bretagnolle, D. Dacunha Castelle, J. L. Krivine, Lois stables et espaces $L^{p}$, Ann. Inst. H. Poincaré Sect. B (N. S.) 2 (1965/1966), 231-259. MR0203757 (34:3605)

[BL] Y. Benyamini and J. Lindenstrauss, Geometric Nonlinear Functional Analysis, Coll. Pub. 48, Amer. Math. Soc., Providence, RI, 2000. MR.1727673 (2001b:46001)

[G] M. Gromov, Asymptotic invariants for infinite groups, Geometric Group Theory, G. A. Niblo and M. A. Roller, eds., Cambridge University Press, Cambridge, 1993. MR:1253544(95m:20041)

[GD] S. Guerre-Delabrière, Types et suites symétriques dans $L^{p}, 1 \leq p<+\infty, p \neq 2$, Israel J. Math. 53 (1986), no. 2, 191-208. MR0845871 (87m:46047)

[JR] W. B. Johnson and N. L. Randrianarivony, $\ell_{p}(p>2)$ does not coarsely embed into a Hilbert space, Proc. Amer. Math. Soc. (to appear).

[MN] M. Mendel, A. Naor, Euclidian quotients of finite metric spaces, Adv. Math. 189 (2004), 451-494. MR2101227 (2005h:54031)

[Ni] E. M. Nikišin, Resonance theorems and expansions in eigenfunctions of the Laplace operator (Russian), Izv. Akad. Nauk SSSR Ser. Mat. 36 (1972), 795-813, English translation: Math. USSR-Izv. 6 (1972), 788-806.

[No] P. Nowak, Coarse embeddings of metric spaces into Banach spaces, Proc. Amer. Math. Soc. 133 (2005), 2589-2596. MR2146202

[R] S. Rolewicz, On a certain class of linear metric spaces, Bull. Acad. Polon. Sci. Sér. Sci. Math. Astrono. Phys. 5 (1957), 471-473. MR0088682(19:562d)

[S] I. J. Schoenberg, Metric spaces and positive definite functions, Trans. Amer. Math. Soc. 44 (1938), 522-536. MR.1501980

Department of Mathematics, Texas A\&M University, College Station, Texas 77843

E-mail address: nirina@math.tamu.edu

Current address: Department of Mathematics, University of Missouri-Columbia, Mathematical Sciences Building, Columbia, Missouri 65211-4100

E-mail address: lova@math.missouri.edu 